\title{
A User-Oriented Language for Specifying Interconnections between Heterogeneous Objects in the Internet of Things
}

DOI:

10.1109/JIOT.2019.2891545

10.1109/JIOT.2019.2891545

\section{Document Version}

Accepted author manuscript

Link to publication record in Manchester Research Explorer

\section{Citation for published version (APA):}

González García, C., Zhao, L., \& García-Díaz, V. (2019). A User-Oriented Language for Specifying

Interconnections between Heterogeneous Objects in the Internet of Things. IEEE Internet of Things Journal . https://doi.org/10.1109/JIOT.2019.2891545, https://doi.org/10.1109/JIOT.2019.2891545

\section{Published in:}

IEEE Internet of Things Journal

\section{Citing this paper}

Please note that where the full-text provided on Manchester Research Explorer is the Author Accepted Manuscript or Proof version this may differ from the final Published version. If citing, it is advised that you check and use the publisher's definitive version.

\section{General rights}

Copyright and moral rights for the publications made accessible in the Research Explorer are retained by the authors and/or other copyright owners and it is a condition of accessing publications that users recognise and abide by the legal requirements associated with these rights.

\section{Takedown policy}

If you believe that this document breaches copyright please refer to the University of Manchester's Takedown Procedures [http://man.ac.uk/04Y6Bo] or contact uml.scholarlycommunications@manchester.ac.uk providing relevant details, so we can investigate your claim.

\section{OPEN ACCESS}




\title{
A User-Oriented Language for Specifying Interconnections between Heterogeneous Objects in the Internet of Things
}

\author{
Cristian González García*, Liping Zhao and Vicente García-Díaz
}

\begin{abstract}
We propose a user-oriented language to enable users to specify interconnections between heterogeneous objects in the Internet of Things (IOT). Based on the idea of the use case specification technique in software engineering, our language provides users with a natural language like syntax to allow them to specify when or under what conditions they want which objects to be connected. To support this language, we have also developed a transformation mechanism that automatically translates users' specification into the source code. We have evaluated this language through an experiment and a survey. The main contributions of this paper are: (1) a simple natural language that enables the users to specify which objects to connect and when, and (2) a transformation mechanism that automatically translates users' specifications into source code and dynamically attaches the code to relevant applications. Our work represents a first step in bringing the loT closer to their users.
\end{abstract}

Index Terms - Internet of Things, Smart Objects, Interoperability, Application Platforms, Service Functions and Management, Service Middleware and Platform, User experience

\section{INTRODUCTION}

$\mathrm{T}_{\mathrm{e}}^{\mathrm{H}}$ HE vision of the Internet of Things (IoT) is to reach out everyday objects in the real world and connect them to the Internet, thus achieving anytime and anyplace connectivity for anyone and anything [1], [2]. According to HIS Markit $^{1}$, by 2020 there will be 17.6 billion physical objects connected to the Internet. If we exclude smartphones, tablets, and computers, Gartner estimates 6.4 billion.

Smart objects, such as smartphones, smartwatches and tablets, play a key role in the IoT vision as they are programmed with intelligent information and communication software. Thus, when connected to sensors, these objects are able to perceive their context and location; with their built-in networking capabilities, they can communicate with each other, access Internet services and interact with people [3]. The same occurs with road vehicles and transportation, which are under the term Internet of Vehicles (IoV), a very important branch of the IoT. For instance, trying to obtain real-time information about the roads, protecting our travel, improving our comfort, the onboard equipment, and vehicular applications [4], [5]. In this way, the main idea is that vehicles can exchange information amongst themselves and with the rest of their environment [6].

- C. González García is with Department of Computer Science, University of Oviedo, Federico García Lorca 18, 33007, Oviedo, Spain. E-mail: gonzalezcristian@uniovi.es.

- L. Zhao is with School of Computer Science, University of Manchester, Oxford Road, Manchester, M13 9PL, United Kingdom, E-mail:

liping.zhao@manchester.ac.uk.

- V. García-Díaz with Department of Computer Science, University of Oviedo, Federico García Lorca 18, 33007, Oviedo, Spain. E-mail: garciavicente@uniovi.es.

* Corresponding author

Copyright (c) 2012 IEEE. Personal use of this material is permitted. However, permission to use this material for any other purposes must be obtained from the IEEE by sending a request to pubs-permissions@ieee.org.
'Conventional objects', such as sewing machines, exercise bikes, electric toothbrushes, washing machines, electricity meters and photocopiers, can have a 'digital makeover', that is, by adding the capabilities of digital objects, to enhance their functionality [3]. With digitalisation (digital objects) and sensors, we can connect both smart and non-smart objects to the Internet [7], make them communicate with each other and create value-added, intelligent applications such as 'Smart Homes' and 'Smart Cities' - the dream of the IoT [8]. However, sometimes, the need of good or special practices is necessary because of the different necessities or limitations of the hardware, like the battery [9], [10] and the energy consumption [11], [12], the low computing power [13], the centralised control of many devices [14], or a standardisation, data management or security, among others [15], [16].

In the IoT, however, things, which can be smart and non-smart [7], are usually diverse, as they are made by different manufacturers, serve different purposes, contain different physical components, use different interface standards, have different communication protocols, embed different software technologies, and so on [17]-[19]. These differences inevitably result in heterogeneous objects that cannot directly communicate [17], [20]. Although global standards on the IoT may ease the heterogeneity problem, creating such standards is currently a major challenge to the IoT [3], [21]-[24]. For example, a recent work [25] focuses on the great number of services that are emerging with the diversity in IoT. Thus, they propose an approach for developing such services based on different aspects such as a technology independent modelling step, an implementation step and a simulation of those services.

A common solution to this problem combines the principle of information hiding and encapsulation with the 


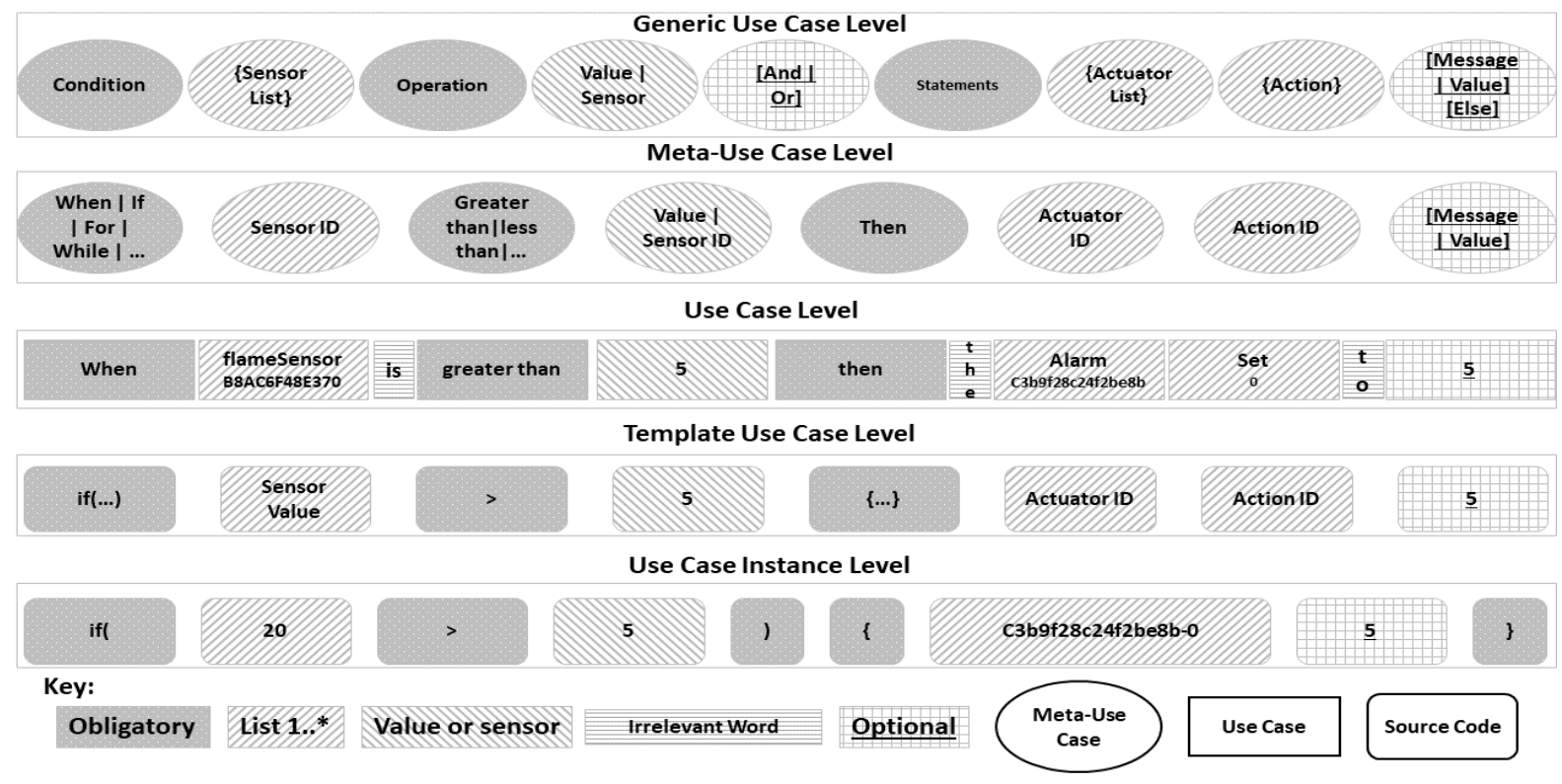

Figure 1 The svntax and structure of MUCSL

concept of Service-Oriented Architecture (SOA). This approach provides each object with a service, which acts as a communicator (i.e., interface) for the object and hides the object details from the client [17]. By using such an approach, objects heterogeneity is hidden from the service consumers, allowing applications to use those objects via standard services.

Yet, the IoT-based applications are dynamic. We quote this example from [17]: 'A device such as a Bluetooth smartphone might become unavailable to a system as soon as it moves out of range. Regarding autonomy concerns, a simple sensor cannot perform its task anymore if its battery is depleted. As a consequence, a system hosting IoT-based pervasive applications must be highly dynamic to manage the devices, which continuously leave or enter the system.' This kind of application, therefore, cannot be fully described beforehand due to the non-deterministic nature of service availability.

While dynamic SOA [26] offers a promising solution to this problem by allowing applications to react to service arrivals and departures according to their environment, developing dynamic applications in this fashion is a specialised job, assuming programming skills and software development knowledge [27]. This assumption seems to be at odds with the vision of the IoT, which inspires pervasive connectivity for anyone and anything at any time and space. Indeed, the IoT-based applications should be pervasive and made for the mass, rather than the few. To take advantage of the IoT, businesses and individuals without professional knowledge should be able to define their own applications and decide when and which of their objects should be connected to provide their desired services.

With this motivation, we propose a user-oriented language to enable users to specify interconnections between heterogeneous objects in the IoT. Based on the idea of the use case driven approach in software engineering [28], our language provides users with a natural language like syntax to allow them to specify when or under what condition they want which objects to be connected. To support this language, we have also developed a transformation mechanism that automatically translates users' specification into the source code.

The main contributions that can be identified from this paper are:

1. A Domain-Specific Language (DSL), very similar to natural language.

2. A DSL that enables the users to specify the interconnection among objects in an easy and simple way without programming.

3. A transformation mechanism that automatically translates users' specifications into source code that includes all the necessary logic.

This contribution can be applied to any field of the IoT. The requirement is a central platform in which the different objects have to be registered.

Although there are already some related works, there is a great absence on DSLs in the IoT and more specifically in the scope of this same work [29]. Thus, our work represents a first step in bringing the IoT closer to their users. Here, users can describe the interconnection that they need between the objects. This interconnection includes conditionals, loops, events (using the different structures), the selection of objects and the messages that you want to send to an object (actions). Thus, using the different structures, users can create the interconnection among objects with a small intelligence or based on different decisions.

We call our specific language MUCSL (Midgar Use Case Specification Language). Midgar is an IoT platform developed in our early work [18]. Midgar is necessary because the objects have to be registered in this platform and have to use the message system described in the platform to create the interconnection, being Midgar our case study to demonstrate this research. We develop MUCSL, which is the main contribution of this work, as a new layer on top of Midgar. The main difference with this previous research is that now, we have developed a textual DSL, which is very close to natural language. This new DSL facilitates the 


\begin{tabular}{|c|c|c|}
\hline \multicolumn{2}{|c|}{ Category } & Words \\
\hline \multirow{2}{*}{ Obligatory } & $\begin{array}{c}\text { Condition } \\
\text { Comparison } \\
\text { Operators }\end{array}$ & $\begin{array}{c}\text { Greater than, more than, less than, lower than, minor than, equal, equal to, greater or equal to, } \\
\text { equal or greater than, less or equal to, equal or less than, more or equal to, equal or more than, } \\
\text { lower or equal to, equal or lower than, minor or equal to, equal or minor than, different from, } \\
\text { set to, }>,<,>=,<=,==, != \\
\text { Then }\end{array}$ \\
\hline \multicolumn{2}{|c|}{ Conjunction } \\
Value
\end{tabular}

Table 1 Keywords of MUCSL

conversion of use cases, according to some rules, to the automated source code generation. This being opposed to the previous research in which users had to learn a graphical DSL to develop the applications.

To continue, the next section details our language and its transformation mechanism. Section 3 presents an experiment on the utility of our approach and evaluates its usability by analysing the experimental results. Section 4 overviews other approaches that are related to our work, and finally, Section 5 concludes the paper by summarising its contributions and outlining future work.

\section{MUCSL: A USER-Oriented LANGUAGE}

In this section, we describe the different parts of this research. First of all, the language syntax of the use cases. Next, how to transform them. After, the Midgar platform, which we have used to this research and how to use MUCSL. Then, the software and hardware used in this research. Lastly, some examples of different uses cases of our proposal.

\subsection{Language Syntax and Structure}

The overall structure of MUCSL consists of five layers (Figure 1). The top layer, Generic Use Case Layer, provides the vocabulary for users to define the sentence structure. This layer consists of six categories of words (see Table 1): obligatory, conjunction, IDs, values, optional, and others. These groups of words can be composed of simple and natural sentences that can be interpreted in a sequential way: First, we have the condition to indicate the beginning of a sentence. Second, we compare the value of a sensor or a list of sensors (to facilitate the use of numerous objects) with a numerical value to establish the truth or falsehood of the condition. Then, we have the option to add 'and' or 'or' in the condition if more sensors are going to be compared. This allows users to create and combine numerous sensors with different conditions in an easy way. The next word is the statement that we use to separate the condition of the clause. The statement contains the list of one or more actuators and the action that the user wants to do on those actuators. Afterwards, we have another optional word, indicating an alternative action either to 'turn on' or 'turn off' the actuator. This research supports different control structures. We have the 'if' when users write 'if' or 'when', the 'else' part of the 'if' when users write 'else' or 'otherwise', and the 'for' and 'while' when users write them respectively. Figure 2 shows a screenshot of the application with a use case written.

Name MidgarTest
Use Case
When the B8AC6F $48 \mathrm{E} 370-\theta$ is greater than 5 then the C3b9f $28 \mathrm{c} 24 \mathrm{f} 2 \mathrm{be} 8 \mathrm{~b}-\theta$
Submit

Figure 2 Screenshot of the application

The second layer, Meta-Use Case Layer, provides possible words that some part of the structure needs. For example, the condition only accepts 'When' or 'If'. The list of sensors needs one or more sensor IDs. The comparison operators can be 'greater than', 'less than', 'equal to', 'greater or equal than', 'less or equal than', 'different from', and so on. The value can be an exact value like a Boolean, a number, a text message, a character, a numeric value, or another sensor to obtain a value. For instance, we could have to turn on the light when two or more photoresistor sensors of our living room have a value smaller than five, or if a value is smaller than the value detected on a photoresistor sensor placed outside the house. The optional value 'and' or 'or' serves us to concatenate more conditions. In this example, we did not use it. After, the statement must be the word 'then'. This word indicates the beginning of the actions that the user wants to do when the conditions are accomplished. The next words are the list of one or more actuator IDs and the action ID that the user wants to activate in these actuators. Finally, we have the optional message or value that the user may have to send to the action. After all, we can type the clause 'else' or 'otherwise' to do an action otherwise, when the normal clause will not accomplish.

MUCSL has a simple lexicon consisting of 'when', 'if', 'for', 'while', 'sensorID', 'then', etc., defined at the MetaUse Case Layer, as Figure 1 shows. MUCSL uses the syntax of conditional statements and allows the users to define 
their intended interconnection through simple conditional statements, such as 'If condition then action' and 'while condition then action'.

The third layer, Use Case Layer, consists of userdefined use case instances. For example, in Figure 1, the statement 'When the B8AC6F48E370 is greater than 5 then the C3b9f28c24f2be8b 0 to 5 ' suggests that the object ID will be used instead of the name because we want to make sure the correct object is called to take the correct action. Users can see the unique IDs of their objects on the Midgar platform using the provided RESTful service. After mapping the IDs to the object names, the statement 'When the $\mathrm{B} 8 \mathrm{AC} 6 \mathrm{~F} 48 \mathrm{E} 370$ is greater than 5 then the C3b9f28c24f2be8b 0 to $5^{\prime}$ will be translated into 'When the flameSensor is greater than 5 then the alarm will be set to $5^{\prime}$.

Figure 3 depicts the described step using MUCSL. We can see that the user writes a use case to use the photoresistor sensor and a sensor of his smartphone. Especially, he wants to turn on the lights when one of these sensors will have a value less than five. Clearly, as we explain before, he has to write this use case using the structure of MUCSL.

\subsection{Use Case Transformation}

The previous section explains how the first three layers of MUCSL can support users to write a use case. This section

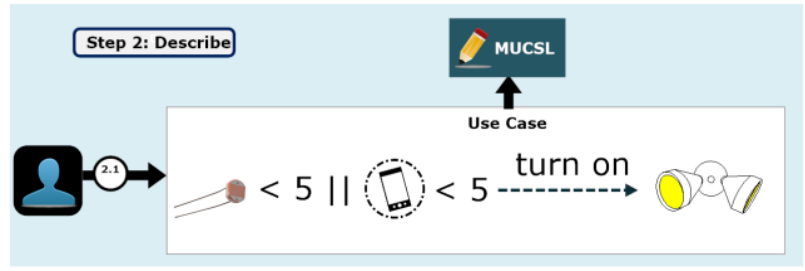

Figure 3 User case description by the user

describes how the last two layers can help transform a use case into the source code.

The Template Use Case Layer takes the text from the Use Case layer as input, removes irrelevant words such as 'the', 'is', 'will', 'to', and so on, and maps relevant data onto the template. In Figure 1, we can see that the obligatory words are transformed into keywords of the programming language, while the other words are settled in their corresponding place.

Finally, the Use Case Instance Layer generates the source code from the template. In our example, we have one sensor, the flame sensor. Then, we have created a use case in which we wrote that when the value of this sensor will be greater than ' 5 ', it will execute an alarm. The value of the flame sensor is read every little time. In Figure 1, we can see that at that exact moment the value is ' 20 '. Maybe, in the next petitions, this value could change or not, it will depend on the distance between the flame and the sensor. In the case of the Actuator ID, we merge the actuator ID with the Action ID to obtain the data that we must send to Midgar for doing the correct query. In this case, we set the value ' 5 ' to the action of that actuator, which has the ID ' 0 '. After that, we obtain the application to interconnect the different objects.

\subsection{Midgar Platform}

Midgar is a cloud-based IoT platform developed in a previous work [18]. This platform has been created accordingly to have a specific platform for implementing and managing the different researches, and allows us to create in an easy way prototypes about the IoT for theses [30] and other research works. For instance, other contributions that have been implemented, used, and tested using Midgar have been presented in other works [31]-[33].

Midgar provides RESTful web services for the registration and management of heterogeneous and ubiquitous objects for IoT applications. Midgar uses an XML-based message system for data exchange and allows the interconnected objects to send or receive the data from each other, if the objects comply with the predefined communication rules [18], [31].

Midgar also possesses basic Artificial Intelligence (AI) [34] capabilities [35] and its intelligence can be improved over time. This part has been facilitating the implementation and demonstration of different researches. Besides, Midgar contains an AI-based decision tree pre-programmed by Midgar users themselves. Thus, based on the input parameter, the platform can then know which object should perform a specific task, make a predefined decision accordingly, and then send it to the recipient object.

Once this object is registered, it can send and receive continuous messages to Midgar in a secure way [32]. These messages can serve to send data from the sensors and the status of this object to Midgar or to receive messages from Midgar about what the receiving object should do. Thus, Midgar is the brain of an entire IoT ecosystem. Then, Midgar is not a contribution but is an important part. Midgar is which manages and interconnects the objects, has the daemon programs that we created using MUCSL, which is the contribution of this paper, and keeps and manages all the information of the objects and daemon programs.

Due to the use of Midgar, this research needs to include the IP of the network to send the different messages to the central server. The server has a database, all the data of the different objects, as it happens in the Midgar IoT platform on which we rely for this research [18]. However, it will be possible to modify the parser to change the part in which includes the IP to use the HTTP protocol to use another protocol.

\subsection{The Process of Using MUCSL}

Using MUCSL is an integral part of the entire IoT application development process, as Figure 4 shows. The process consists of four simple steps: (1) Register - registering the objects to the IoT platform, (2) Describe-describing their interconnection in use cases, (3) Transform - transforming the use cases into source code, and finally (4) Run-executing the IoT applications through the interconnected objects.

These steps, which have been implemented as web services on Midgar, are described in detail as follows.

Register: This is the original service provided by Midgar. Users register their objects on the Midgar platform by providing the information of the objects in the XML format. This information includes the identifications of the 
objects, the identifications of the sensors attached to the objects, and the actions that can be performed by the objects. Besides, when the program is generated for each object and is uploaded to the object, it contains all the logic to communicate it with the platform and with other objects. In this research, every Midgar user can access to any object that is registered in the server. However, the platform implements a security system to send secure messages between objects [32].

Describe: Users describe when and under what conditions they want to use which objects in a use case using MUCSL (Figure 4). The syntax of MUCSL is similar to the syntax of the English language. Here, the user uses the created DSL, MUCSL, to define the use case that describes the desired interconnection. To do this, users write, using natural language, what he wants to create following a series of guidelines, which are shown in Figure 1. For instance, if they want to create a system to read the information of a sensor and send an alarm to another object, they have to write this according to the syntax which was defined and explained in Figure 1. After this, all this information is saved in an XML to send to the transform step.

Transform: The use case is sent to the Transformer (Figure $4: 3.1$, which reads the use case, analyses it, and creates the corresponding source code according to the use case description (Figure 4: 3.2). In this step, the transformer receives the use case, which was defined by the user, in XML format with only the needed information but without irrelevant words or punctuation characters. The transformer parses all the received information and creates an active process that contains how the interconnection of objects is. The transformer is a Java application, which is a part of the transformer situated in Step 3 (Figure 4), which reads the information of the use case. This information is read and inserted in a template that has the general information of

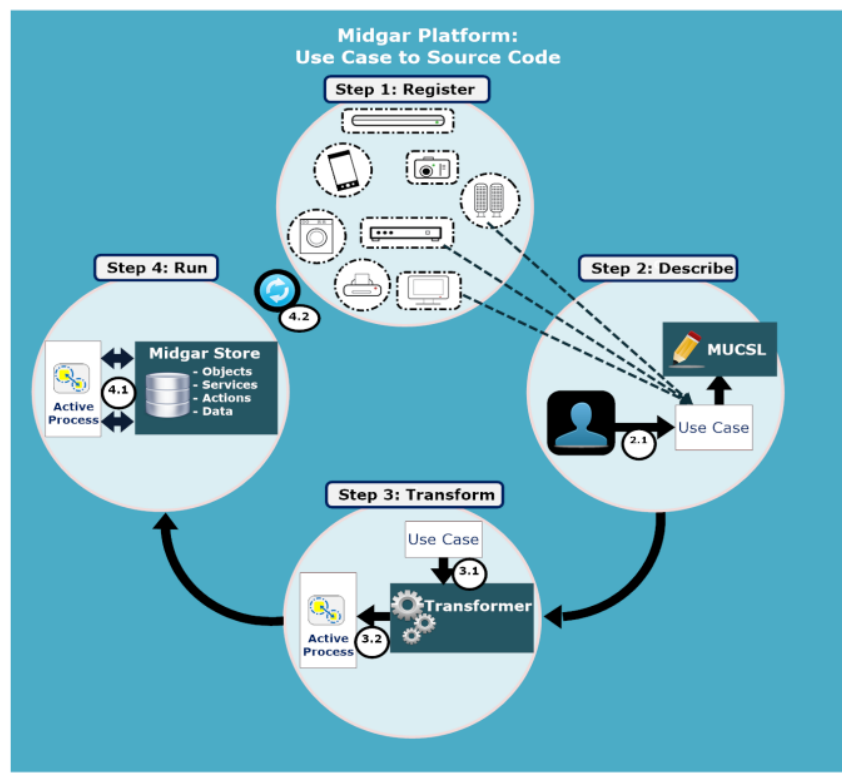

Figure 4 The proposed approach supports the development of interconnection software through a lifecycle process, consisting of a the daemon: access to the databases (IP, password, queries), and the architecture and basic structures of the source code using a Visitor pattern. Then, the Java application goes iterating by the different nodes, which have obtained from the use case, and change the part that it needs in the template. Then, the Java application completes the template with the information of the specific use cases which are contained and provided in the XML that was received in this layer: objects ID, Server IP, which data of the objects have to compare, how to compare it (greater than, less than, and so on), and how to use that data. This application also contains the needed libraries to access to the database and parser the XML messages.

Run: In this step, an Active Process will execute the incoming message sent from the Transformer (Figure 4: 4.1). The message instructs the Active Process how to connect different registered objects. When the transformer creates the active process, the Active Process can be run in Midgar platform or in our own computer, because this process will send all the information to Midgar. The Active Process is continuing consulting the database of Midgar because this platform is which manages and keeps all the information of all the registered objects. Especially, it is consulting the data of the objects that are presented in the use case that the user defined. Then, the Active Process reads the values of these objects and tests the condition. If the condition does not accomplish, the active process does not do anything. In the case that the condition is accomplished, then, the Active Process sends a query to Midgar to set the action that the user defined in the use case, and set all the needed messages and values for that application. In this way, the platform can send that information to that object Meanwhile, all the registered object in Midgar are sending the values of their sensors to the platform. These values are managed and read for the Active Process that was created to solve and manage the corresponding use case.

\subsection{Used Software and Hardware}

To develop this research work it is required the use of different types of software and hardware components that are shown in Table 2.

\begin{tabular}{|c|c|}
\hline \multicolumn{2}{|r|}{ The IoT Midgar platform } \\
\hline Ruby & 2.5.1p57 \\
\hline Rails & 5.2 .1 \\
\hline Thin Web Server & 1.7 .2 \\
\hline MariaDB & 10.1.29-MariaDB-6 \\
\hline \multicolumn{2}{|l|}{ MUCSL } \\
\hline HTML & 5 \\
\hline PHP & 7.2.10 \\
\hline JavaScript & Standard without external libraries \\
\hline \multicolumn{2}{|r|}{ Application Generator } \\
\hline Java & 10 \\
\hline \multirow[t]{2}{*}{ Arduino } & RXTXcomm.jar \\
\hline & $\begin{array}{l}\text { HTTPComponents of Apache Software } \\
\text { Foundation }\end{array}$ \\
\hline Android & $\begin{array}{l}\text { HTTPComponents of Apache Software } \\
\text { Foundation }\end{array}$ \\
\hline \multicolumn{2}{|r|}{ Dedicated Server } \\
\hline $\begin{array}{l}\text { Raspberry Pi } 2 \\
\text { Model B }\end{array}$ & Raspbian Jessie 4.9 .75 v7+ \\
\hline
\end{tabular}




\begin{tabular}{|l|l|}
\hline $\begin{array}{l}\text { 3 Android } \\
\text { Smartphone and 1 } \\
\text { emulator }\end{array}$ & One Android emulator with Android 8.1 \\
\cline { 2 - 2 } & Moto 5GS Plus with Android 7.1 \\
\cline { 2 - 2 } & Nexus 4 with Android 5.1.1 \\
\cline { 2 - 2 } Arduino & Motorola with Android 2.2.2 \\
\hline Sensors & $\begin{array}{l}\text { The SMD based on ATmega328 } \\
\text { sistor, the temperature and humidity sen- } \\
\text { sor DHT11, and the flame detector KY026 }\end{array}$ \\
\hline Actuators & $\begin{array}{l}\text { A speaker, a servomotor, a DC motor, and } \\
\text { different LEDs }\end{array}$ \\
\hline
\end{tabular}

Table 2 Used software and hardware

We have chosen Ruby on Rails to develop the platform in the server side in the first iteration, in 2013, because the facilities that the Rails framework gives us to maintain and deploy applications, and the facilities to build all the scaffold. Besides, Rails is one of the most popular framework for web development [36]. Currently, in GitHub [37], Ruby is the fifth General-Programming Language (GPL) more used and in the Tiobe Index [38] was in the eight position in 2016.

HTML, CSS, and JavaScript are used to create the web interfaces because they are the industry standard that were created to this task, and JavaScript is the most popular GPL to program in the client side according to both references [37], [38].

PHP has chosen to develop the new web service to separate from Rails. PHP has a lot to resources because it is a GPL which was designed and is maintained for web applications. Furthermore, PHP is the most popular web scripting GPL [39] for years [38] and it is used in several famous webs. Besides, in last years, Facebook has done a lot of effort in improving this GPL. First of all, with the optimisation of HipHop to translate PHP code to C++ code [40]. Secondly, creating a new GPL called Hack, which is a variant of PHP [39], [41], to solve the dynamic typing. Finally, with the HHVM virtual machine that can execute PHP and Hack code.

Java has chosen to develop the parser, which is on the server side, because all the facilities and good performance that Java has for this type of tasks.

Then, we have Ruby on Rails as a central server, Java to parser all the XML files, PHP as a web service to this research, and HTML, CSS, and JavaScript to develop all the graphical DSL. In each moment, we try to develop the research with the last and 'best' technologies for each task.

\subsection{Use Case Examples}

In this section, we show different examples to demonstrate the capacity of the DSL:

- Combination of two sensors that have to be equal than five and another one than has to be less than 20 to activate the action that requires or needs, or may be allowed sending a not default value, which is 100 in this case. This use case could be a system to automate the heather based on temperature sensors, a sound alarm or an automatic door that has two proximity sensors to cover range defined in meters.

- When the B8AC6F48E370-0, B8AC6F48E3701 are equal to 5 and B8AC6F48E370-1 is less than 20 then the C3b9f28c24f2be8b-0 to 100 .
- A sensor that has to be greater than 5 and a second sensor that has to be less than 20. If both cases are accomplished, then the application activates two actions, which do not need any value. This use case could be an alarm system with two actions like sound and pictures, VoIP calls or text messages, or a system to ventilate a room according to two gas sensors.

- When the B8AC6F48E370-0 is greater than 5 and B8AC6F48E370-1 is less than 20 then the C3b9f28c24f2be8b-0 and D4az78t31y7ghu8p0.

- When the values of the three sensors are greater than 28 , then the first and second actions of the second device will be launched. In all other cases, the actions 0 and 1 of the third device will be run. This use case could be matched with distributed sensors along a place to detect a determinate measure that could be dangerous or uncomfortable gases $\left(\mathrm{CO}, \mathrm{CO}_{2}, \mathrm{H}_{2}\right.$, etc.), or temperature, to open different windows or doors, activate an alarm, start an extractor, other ones, or various of them.

- If B8AC6F48E370-0, B8AC6F48E370-2, B8AC6F48E370-3 are greater than 28 then the C3b9f28c24f2be8b-0, C3b9f28c24f2be8b-1 else the Z7k194iop22ns89e-0 and Z7k194iop22ns89e -1.

- In case we want to increment or do a repetitive task, we can use the loop to launch one or more actions when one or more sensors reach the defined parameter/s.

- Meanwhile, the B8AC6F48E370-1 is less than 10 then the Z7k194iop22ns89e-0

- This use case can be a loop that is executing meanwhile the temperature is greater than $30^{\circ} \mathrm{C}$ and the humidity is greater than $80 \%$ to call two services to increment the air conditioning and the dehumidifier until the sensors detect again a normal temperature $\left(<30^{\circ} \mathrm{C}\right.$ and $<80 \%$ humidity). In the other case, the data will be sent to a $\log$ web service.

- While the B8AC6F48E370-0 is greater than 30 and the B8AC6F48E370-1 is greater than 80 then the Z7k194iop22ns89e-1 and Z7k194iop22ns89e-2 else Z7k194iop22ns89e-0

The examples used in this paper are the most common ones that people use at home or in industry, but the proposed DSL of this research supports the use of any other device or web service that uses the Midgar platform and their systems. Other devices that can be supported can use RFID tags to detect when they are used by someone or something, any type of sensor or actuator that you can connect to an Arduino, smartphones, web services, a robot that has Internet connection to move according to other sensors or devices, or any other device with Internet connection and the possibility of parsing an XML.

\section{Evaluation and Results}

This section first describes the methodology used in the evaluation of our prototype and then discusses the evaluation results. The evaluation was divided into two phases. 
The first phase is gathering quantitative data from the evaluation made to the participants, which consists of three tests. The second phase is a survey made by the participants after testing the first phase, which gives us a qualitative assessment.

\subsection{Methodology}

The main objective of this evaluation has been to validate the initial hypothesis, for which the presented objectives must be fulfilled. To achieve this, MUCSL has been created, a language close to natural language that allows, by complying with a series of rules, to transform the use cases written by the participants into the final application that will interconnect objects. Twenty-one participants have taken part in the evaluation with the following features:

- $95.2 \%$ had heard about the IoT.

- $38.1 \%$ had never worked with the IoT before.

- $81 \%$ had heard about Smart Objects.

- $38.1 \%$ had never worked with Smart Objects before.

- $90.5 \%$ were familiar with use cases.

Notwithstanding, the only thing that the users have to know is the rules of MUCSL. MUCSL and an introduction to the IoT, Smart Objects and the problem and solution of the research were explained before the experiment to each participant independently.

To validate the hypothesis, two phases have been developed so that the first one obtains quantitative data and the second one qualitative data to evaluate it correctly:

- Phase 1: in this first phase, three tests have been proposed to the participants. These tests have been three possible use cases of interconnection of objects that have been written using MUCSL. This phase corresponds to the quantitative evaluation.

- Phase 2: after completing phase 1, the 21 participants had to answer a survey, putting together 17 statements, using the 5-point Likert scale [42]. The survey contains statements about MUCSL and what was done in the first phase, so that the participants should state how they agree with each of the statements. This gives the qualitative data to the evaluation.

The background of the participants is as follows:

- The $95.2 \%$ of users had heard about the IoT but only the $38.1 \%$ had worked with the IoT.

- The $81 \%$ of the users had heard about Smart Objects but only the $38.1 \%$ had worked with them.

- The $90.5 \%$ had heard about use cases and the $81 \%$ had worked with them.

\subsubsection{Phase 1}

In this first phase, the participants had to perform three tasks using MUCSL, each task being independent of the others. The three tasks were sent to the user to facilitate understanding, being the first task easier and the second and third similar, although more difficult.

During the tests, quantitative data has been acquired regarding the use of the editors. For example, the time in seconds that each participant needed to make the application, the displacement, measured in centimetres, with the mouse and the clicks with the right and left mouse buttons. To measure this data the tool Mousotron [43] has been used.

To create the three tasks, we though in examples that can be used thoroughly by almost all people in normal and typical tasks. However, as we have seen in section 2.6, we can use this DSL to more complex tasks.

The first task has been to create an application that interconnects an Arduino with a smartphone. The purpose is for this application to use the Arduino's Flame Sensor to detect fire. This first example is based on the common use of a fire detector for Smart Homes, in the Industrial IoT (IIoT), Smart Towns, Smart Cities, or in Smart Earth.

Thus, when it marks a value of 50 or less, the application should send a notification to the smartphone with the message 'Fire'. The sensor identifier is 'B8AC6F48E370-0' and the smartphone identifier is ' $\mathrm{C} 3 \mathrm{~b} 9 \mathrm{f} 28 \mathrm{c} 24 \mathrm{f} 2 \mathrm{be} 8 \mathrm{~b}-0$ '. A possible solution of the first task is shown in Source Code 1, where possible optional words of the phrase are shown in square brackets ('[' and ' $]^{\prime}$ ).

If [the] B8AC6F48E370-0 [is] greater than 49 then C3b9f28c24f2be8b-0 [to] 'fire'

\section{Source Code 1. The solution for the first task}

The second task has been to develop an application that will interconnect the Arduino with a smartphone and a light. The application must use the Arduino photoresistor and the smartphone to detect whether the light needs to be turned on. This second example is based on turning on/off the lights of a place automatically using a permanent Arduino with a photoresistor and using the smartphone as a mobile sensor. Especially, this application can be used in Smart Homes and in the IIoT because they are indoor places. For instance, in [44], we can see different services of the IoT and IIoT in which this could be applied, like health, education, agriculture, logistic, etc.

If the Arduino sensor drops to a value of 30 or less and the smartphone to a value of 20 or less, the application must send the command to turn on the light, otherwise, the light must be turned off. The identifiers of the Arduino and smartphone sensors are 'B8AC6F48E370-0' and 'C3b9f28c24f2be8b-0' respectively. Meanwhile, the identifiers of the actions to turn on and off the light are 'D4az78t31y7ghu8p-0' and 'D4az78t31y7ghu8p-1' for each case. A possible solution to the second task is shown in Source Code 2.

When [the] B8AC6F48E370-0 [is] equal or less than
30 and C3b9f28c24f2be8b-0 [is] equal or less than
20 then [the] D4az78t31y7ghu8p-0 else [the]
D4az78t31y7ghu8p-1

Source Code 2 The solution for the second task

In the third task, the participants had to develop an application that interconnects the Arduino and a fan. Thus, by using the Arduino temperature sensor the application will be able to change the fan speed of movement. This last task is the classical example based on the use of a temperature system to automate a fan in Smart Homes. 
If the Arduino sensor reaches a value of $25^{\circ} \mathrm{C}$ or more, the application will add a speed point to the fan for each degree of difference. Otherwise, the application will turn off the fan. The identifiers are 'B8AC6F48E370-1', 'Z7k194iop22ns89e-0' and 'Z7k194iop22ns89e-1' for the Arduino sensor and the increase and decrease of the speed of the fan. A possible solution to the third task is shown in
Source Code 3.

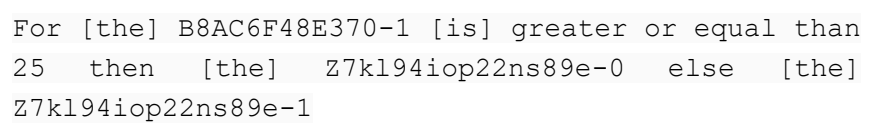

Z7k194iop22ns89e-1

\section{Source Code 3 The solution for the third task}

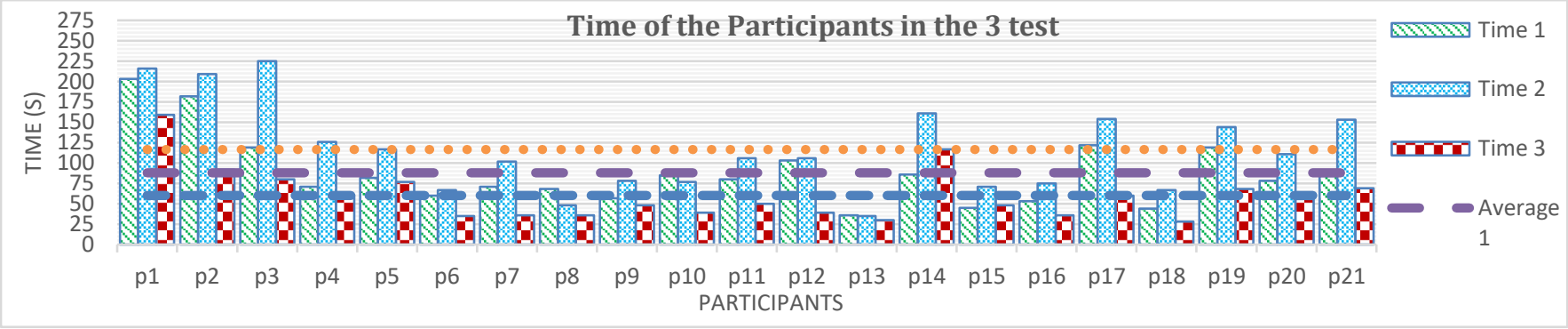

Figure 5 Time required by each participant in each task along with the overall average

\subsubsection{Phase 2}

In the second phase, the qualitative part of MUCSL was evaluated to obtain the opinion of the participants and to know what they think on this research. To do this survey, the 5-point Likert scale has been used as an evaluation method because it is a widely used method in the field of software engineering to obtain information effectively to support decision making [45].

When using the 5-point Likert scale, containing a total of 17 statements, 5 possible responses are offered: 1 as Totally Disagree, 2 as Disagree, 3 as Neutral, 4 as Agree, and 5 as Totally agree.

The participants always performed this survey after completing phase 1 , anonymously and without help. This survey contains statements about MUCSL, its possibilities and possible impact on the IoT and Smart Objects, offering an interconnection between the two. Table 3 contains the statements.

\begin{tabular}{cl}
\hline$\#$ & \multicolumn{1}{c}{ Declarations } \\
\hline D1 & $\begin{array}{l}\text { You can understand the structure of the elements and their } \\
\text { role in the application creation process. }\end{array}$ \\
D2 & $\begin{array}{l}\text { This tool offers a useful help to interconnect heterogeneous } \\
\text { objects. }\end{array}$ \\
D3 & The syntax is clear, easy, and natural. \\
D4 & $\begin{array}{l}\text { This solution offers a fast way for you to specify how you } \\
\text { wish to connect your devices. }\end{array}$ \\
D5 & $\begin{array}{l}\text { This solution provides assistance to interconnect objects. } \\
\text { The way to create interconnections using this language is } \\
\text { D6 }\end{array}$ \\
D7 & $\begin{array}{l}\text { The language does not require the user to use complex pro- } \\
\text { gramming skills, as in traditional application development. }\end{array}$ \\
The syntax includes enough elements and functionality for \\
the user to create a wide range of interconnections to ob- \\
jects.
\end{tabular}

\begin{tabular}{cl}
\hline$\#$ & \multicolumn{1}{c}{ Declarations } \\
\hline D11 & $\begin{array}{l}\text { This language could be used to simplify the classic devel- } \\
\text { opment process of software applications in other areas (ed- } \\
\text { ucation, video games, and so on). }\end{array}$ \\
D12 & $\begin{array}{l}\text { The use of this language reduces the complex development } \\
\text { for this type of applications. }\end{array}$ \\
D13 & $\begin{array}{l}\text { This syntax provides an easy and intuitive way to intercon- } \\
\text { nect devices }\end{array}$ \\
D14 & $\begin{array}{l}\text { The syntax of the use cases and the role of the use cases in } \\
\text { application creation process are clear. }\end{array}$ \\
D15 & $\begin{array}{l}\text { The user makes less mistakes if he uses this language than } \\
\text { if they programmes }\end{array}$ \\
D16 & $\begin{array}{l}\text { The user works quicker and more effective if he uses this } \\
\text { language than if they programmes }\end{array}$ \\
D17 & This language can be useful \\
\hline
\end{tabular}

Table 3 Statements in the MUCSL survey

\subsection{Results}

This section analyzes and discusses the results obtained in the two phases. Section 3.2.1 provides a quantitative analysis of the results obtained by performing the three tasks with MUCSL. The qualitative results obtained from the survey are presented in Section 3.2.2.

\subsubsection{Results of Phase 1}

In this phase, the time of each participant has been taken while performing the three tasks required. This time is shown in Figure 5, which contains the time of each participant in each task and the overall average among all participants to perform each task.

Analyzing this graph, the following interpretations can be suggested:

- All participants took a little longer to perform the second tasks compared to the first, with the exceptions of P8, P10 and P13.

- The third task, with a difficulty like the second one, took less than the two previous tasks, except P14 and P15.

- In general terms, it seems to provide a rapid learning curve due to the decrease in the average of the third task compared to the previous two. The 
increment in the second task seems to be due to the increase in the difficulty of the requested task.

\subsubsection{Results of Phase 2}

Table 4 shows the responses of each participant for each survey statement.

\begin{tabular}{|l|l|l|l|l|l|l|l|l|l|l|l|l|l|l|l|l|l|}
\hline & $\mathbf{D}$ & $\mathbf{D}$ & $\mathbf{D}$ & $\mathbf{D}$ & $\mathbf{D}$ & $\mathbf{D}$ & $\mathbf{D}$ & $\mathbf{D}$ & $\mathbf{D}$ & $\mathbf{D}$ & $\mathbf{D}$ & $\mathbf{D}$ & $\mathbf{D}$ & $\mathbf{D}$ & $\mathbf{D}$ & $\mathbf{D}$ & $\mathbf{D}$ \\
& & $\mathbf{2}$ & $\mathbf{3}$ & $\mathbf{4}$ & $\mathbf{5}$ & $\mathbf{6}$ & $\mathbf{7}$ & $\mathbf{8}$ & $\mathbf{9}$ & $\mathbf{1}$ & $\mathbf{1}$ & $\mathbf{1}$ & $\mathbf{1}$ & $\mathbf{1}$ & $\mathbf{1}$ & $\mathbf{1}$ & $\mathbf{1}$ \\
\hline P01 & 3 & 5 & 5 & 4 & 5 & 4 & 4 & 5 & 5 & 5 & 5 & 5 & 4 & 5 & 4 & 4 & 5 \\
\hline P02 & 4 & 5 & 4 & 4 & 4 & 4 & 4 & 4 & 5 & 5 & 5 & 4 & 4 & 5 & 5 & 4 & 5 \\
\hline P03 & 3 & 4 & 3 & 4 & 4 & 3 & 5 & 4 & 4 & 4 & 4 & 4 & 5 & 5 & 5 & 5 & 4 \\
\hline P04 & 4 & 3 & 5 & 3 & 3 & 5 & 5 & 3 & 5 & 4 & 4 & 4 & 5 & 5 & 4 & 4 & 5 \\
\hline P05 & 5 & 5 & 5 & 5 & 5 & 5 & 5 & 5 & 5 & 5 & 5 & 5 & 5 & 5 & 5 & 5 & 5 \\
\hline P06 & 4 & 4 & 4 & 4 & 4 & 5 & 5 & 4 & 5 & 5 & 5 & 5 & 5 & 4 & 5 & 5 & 5 \\
\hline P07 & 4 & 5 & 5 & 4 & 5 & 5 & 4 & 3 & 5 & 4 & 5 & 3 & 4 & 4 & 5 & 5 & 5 \\
\hline P08 & 5 & 4 & 4 & 4 & 5 & 4 & 3 & 3 & 4 & 4 & 2 & 4 & 4 & 5 & 5 & 4 & 5 \\
\hline P09 & 4 & 5 & 4 & 4 & 3 & 4 & 5 & 4 & 4 & 4 & 3 & 4 & 4 & 4 & 3 & 4 & 3 \\
\hline P10 & 5 & 5 & 4 & 5 & 5 & 4 & 3 & 4 & 5 & 4 & 2 & 4 & 2 & 5 & 4 & 5 & 5 \\
\hline P11 & 4 & 4 & 4 & 4 & 4 & 4 & 4 & 4 & 4 & 4 & 3 & 4 & 4 & 4 & 4 & 4 & 4 \\
\hline P12 & 5 & 5 & 4 & 5 & 5 & 4 & 4 & 5 & 5 & 4 & 5 & 5 & 5 & 4 & 5 & 5 & 5 \\
\hline P13 & 4 & 5 & 5 & 5 & 4 & 5 & 5 & 3 & 5 & 5 & 5 & 5 & 5 & 4 & 4 & 5 & 5 \\
\hline P14 & 5 & 4 & 3 & 4 & 4 & 4 & 2 & 3 & 3 & 3 & 4 & 3 & 4 & 3 & 3 & 3 & 4 \\
\hline P15 & 4 & 5 & 4 & 5 & 5 & 5 & 4 & 4 & 4 & 4 & 2 & 4 & 4 & 4 & 4 & 4 & 4 \\
\hline P16 & 5 & 5 & 5 & 5 & 5 & 5 & 4 & 4 & 4 & 4 & 5 & 5 & 5 & 5 & 5 & 5 & 4 \\
\hline P17 & 5 & 5 & 4 & 5 & 5 & 4 & 4 & 4 & 5 & 5 & 4 & 4 & 5 & 5 & 5 & 4 & 4 \\
\hline P18 & 5 & 5 & 5 & 5 & 5 & 4 & 4 & 4 & 5 & 5 & 5 & 5 & 5 & 5 & 5 & 5 & 5 \\
\hline P19 & 5 & 4 & 4 & 5 & 4 & 5 & 4 & 4 & 4 & 4 & 3 & 3 & 5 & 5 & 3 & 4 & 4 \\
\hline P20 & 5 & 5 & 4 & 5 & 5 & 5 & 4 & 5 & 5 & 5 & 4 & 5 & 5 & 5 & 5 & 5 & 5 \\
\hline P21 & 4 & 5 & 4 & 5 & 4 & 5 & 4 & 4 & 5 & 5 & 5 & 5 & 4 & 4 & 4 & 4 & 5 \\
\hline
\end{tabular}

Table 4 Participant responses for each MUCSL statement

Table 5 contains the global descriptive statistics of the MUCSL survey evaluation. This table shows the minimum, the first quartile, the median or second quartile, the third quartile, the maximum, the range between quartiles and the mode of each of the 17 statements of the survey.

\begin{tabular}{|c|c|c|c|c|c|c|c|c|c|c|c|c|c|c|c|c|c|}
\hline & $\mathbf{D}$ & $\mathbf{D}$ & $\mathbf{D}$ & $\mathbf{D}$ & $\mathbf{D}$ & $\mathbf{D}$ & $\mathbf{D}$ & $\mathbf{D}$ & $\mathbf{D}$ & $\mathbf{D}$ & $\mathbf{D}$ & $\mathbf{D}$ & $\mathbf{D}$ & $\mathbf{D}$ & $\mathbf{D}$ & $\mathbf{D}$ & $\mathbf{D}$ \\
\hline & $\mathbf{1}$ & $\mathbf{2}$ & $\mathbf{3}$ & $\mathbf{4}$ & $\mathbf{5}$ & $\mathbf{6}$ & $\mathbf{7}$ & $\mathbf{8}$ & $\mathbf{9}$ & $\mathbf{1}$ & $\mathbf{1}$ & $\mathbf{1}$ & $\mathbf{1}$ & $\mathbf{1}$ & $\mathbf{1}$ & $\mathbf{1}$ & $\mathbf{1 7}$ \\
\hline Min & 3 & 3 & 3 & 3 & 3 & 3 & 2 & 3 & 3 & 3 & 2 & 3 & 2 & 3 & 3 & 3 & 3 \\
\hline $\begin{array}{c}\text { Quar- } \\
\text { tile1 }\end{array}$ & 4 & 4 & 4 & 4 & 4 & 4 & 4 & 4 & 4 & 4 & 3 & 4 & 4 & 4 & 4 & 4 & 4 \\
\hline Median & 4 & 5 & 4 & 5 & 5 & 4 & 4 & 4 & 5 & 4 & 4 & 4 & 5 & 5 & 5 & 4 & 5 \\
\hline $\begin{array}{c}\text { Quar- } \\
\text { tile3 }\end{array}$ & 5 & 5 & 5 & 5 & 5 & 5 & 5 & 4 & 5 & 5 & 5 & 5 & 5 & 5 & 5 & 5 & 5 \\
\hline Max & 5 & 5 & 5 & 5 & 5 & 5 & 5 & 5 & 5 & 5 & 5 & 5 & 5 & 5 & 5 & 5 & 5 \\
\hline Range & 2 & 2 & 2 & 2 & 2 & 2 & 3 & 2 & 2 & 2 & 3 & 2 & 3 & 2 & 2 & 2 & 2 \\
\hline $\begin{array}{c}\text { Inter. } \\
\text { range }\end{array}$ & 1 & 1 & 1 & 1 & 1 & 1 & 1 & 0 & 1 & 1 & 2 & 1 & 1 & 1 & 1 & 1 & 1 \\
\hline Mode & 5 & 5 & 4 & 5 & 5 & 4 & 4 & 4 & 5 & 4 & 5 & 5 & 5 & 5 & 5 & 4 & 5 \\
\hline
\end{tabular}

\section{Table 5 MUCSL global descriptive statistics}

Figure 6 shows the data for each question represented in a box in the diagram.

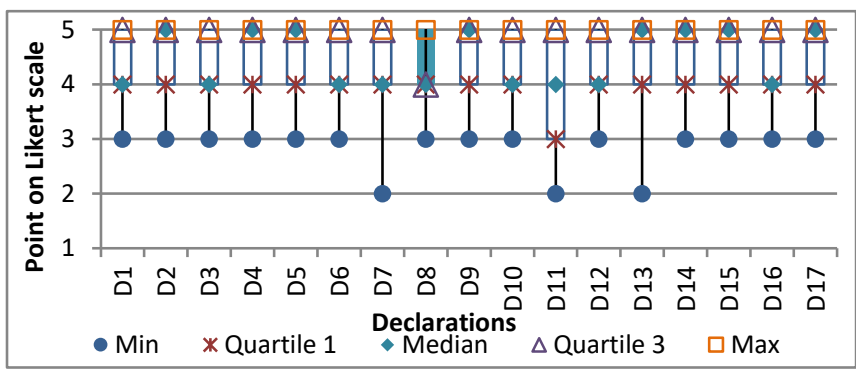

Figure 6 Global Box Diagram for each MUCSL statement

Thus, from the data collected and shown in Table 5 and Figure 6 the following interpretations can be suggested:
- All statements have a maximum of 5, which indicates that at least 1 person has fully agreed with each one.

- All statements, except D7, D11 and D13, have a minimum of 3 , indicating that in these statements the worst case has been 'neutral'.

- D7, D11 and D13 have the smallest minimum as 'disagreement'.

- D2, D4, D5, D9, D13, D14, D15 and D17 have the highest median. From this, we can deduce that most of the participants are totally in agreement with these statements.

- D7, D11 and D13 have a range of 3, which indicates that there is a great diversity of opinions on these statements. The rest of the statements have a range of 2, which indicates that the participants have a similar basic opinion on those statements.

- According to the mode, we can see that D1, D2, D4, D5, D9, D11, D12, D13, D14, D15 and D17 have a mode of 5, which indicates that most of the chosen answers have been 'totally agree'. The rest have a mode of 4 , indicating that the next most chosen answer has been 'agree'.

- D11 with a range of 3, a minimum of 2, a median of 4 , a mode of 5 , an interquartile range of 2 and a maximum of 5 , is the most dubious statement, although the most repeated answer was 'Totally agree'. On the other hand, looking at D7, which has a range of 3 , a minimum of 2 , a median of 4 , a mode of 4 , an interquartile range of 1 and a maximum of 5 , is the worst-valued statement. However, D13 with a range of 3, a minimum of 2, a median of 5 a mode of 5 , an interquartile range of 1 and a maximum of 5 is very well valued, although there are some participants that do not agree.

- $\quad$ D8 is one of the worst rated statements because it has a range of 2 , a minimum of 3 , a median of 4 , is the only one with the quartile 3 in 4 , an interquartile range of 0 and a mode of 4 . This indicates that most participants are only in agreement with it and have a very similar opinion among them since it is the statement with a lower interquartile range.

Table 6 shows the different frequencies obtained from the survey for each statement based on the answers chosen by the participants. This table contains the breakdown of each question to show the number of votes for each decision and their corresponding percentage.

\begin{tabular}{|c|c|c|c|c|c|c|}
\hline $\begin{array}{l}\text { State- } \\
\text { ment }\end{array}$ & & $\begin{array}{l}\text { Strongly } \\
\text { Disagree }\end{array}$ & Disagree & Neutral & Agree & $\begin{array}{l}\text { Strongly } \\
\text { Agree }\end{array}$ \\
\hline \multirow[t]{2}{*}{ D1. } & $\#$ & 0 & 0 & 2 & 9 & 10 \\
\hline & $\%$ & $0 \%$ & $0 \%$ & $10 \%$ & $43 \%$ & $48 \%$ \\
\hline \multirow[t]{2}{*}{ D2. } & $\#$ & 0 & 0 & 1 & 6 & 14 \\
\hline & $\%$ & $0 \%$ & $0 \%$ & $5 \%$ & $29 \%$ & $67 \%$ \\
\hline \multirow[t]{2}{*}{ D3. } & $\#$ & 0 & 0 & 2 & 12 & 7 \\
\hline & $\%$ & $0 \%$ & $0 \%$ & $10 \%$ & $57 \%$ & $33 \%$ \\
\hline \multirow[t]{2}{*}{ D4. } & $\#$ & 0 & 0 & 1 & 9 & 11 \\
\hline & $\%$ & $0 \%$ & $0 \%$ & $5 \%$ & $43 \%$ & $52 \%$ \\
\hline \multirow[t]{2}{*}{ D5. } & $\#$ & 0 & 0 & 2 & 8 & 11 \\
\hline & $\%$ & $0 \%$ & $0 \%$ & $10 \%$ & $38 \%$ & $52 \%$ \\
\hline \multirow[t]{2}{*}{ D6. } & $\#$ & 0 & 0 & 1 & 10 & 10 \\
\hline & $\%$ & $0 \%$ & $0 \%$ & $5 \%$ & $48 \%$ & $48 \%$ \\
\hline
\end{tabular}




\begin{tabular}{|c|c|c|c|c|c|c|}
\hline \multirow[t]{2}{*}{ D7. } & \# & 0 & 1 & 2 & 12 & 6 \\
\hline & $\%$ & $0 \%$ & $5 \%$ & $10 \%$ & $57 \%$ & $29 \%$ \\
\hline \multirow[t]{2}{*}{ D8. } & \# & 0 & 0 & 5 & 12 & 4 \\
\hline & $\%$ & $0 \%$ & $0 \%$ & $24 \%$ & $57 \%$ & $19 \%$ \\
\hline \multirow[t]{2}{*}{ D9. } & $\#$ & 0 & 0 & 1 & 7 & 13 \\
\hline & $\%$ & $0 \%$ & $0 \%$ & $5 \%$ & $33 \%$ & $62 \%$ \\
\hline \multirow[t]{2}{*}{ D10. } & \# & 0 & 0 & 1 & 11 & 9 \\
\hline & $\%$ & $0 \%$ & $0 \%$ & $5 \%$ & $52 \%$ & $43 \%$ \\
\hline \multirow[t]{2}{*}{ D11. } & \# & 0 & 3 & 3 & 5 & 10 \\
\hline & $\%$ & $0 \%$ & $14 \%$ & $14 \%$ & $24 \%$ & $48 \%$ \\
\hline \multirow[t]{2}{*}{ D12. } & $\#$ & 0 & 0 & 3 & 9 & 9 \\
\hline & $\%$ & $0 \%$ & $0 \%$ & $14 \%$ & $43 \%$ & $43 \%$ \\
\hline \multirow[t]{2}{*}{ D13. } & $\#$ & 0 & 1 & 0 & 9 & 11 \\
\hline & $\%$ & $0 \%$ & $5 \%$ & $0 \%$ & $43 \%$ & $52 \%$ \\
\hline \multirow[t]{2}{*}{ D14. } & $\#$ & 0 & 0 & 1 & 8 & 12 \\
\hline & $\%$ & $0 \%$ & $0 \%$ & $5 \%$ & $38 \%$ & $57 \%$ \\
\hline \multirow[t]{2}{*}{ D15. } & $\#$ & 0 & 0 & 3 & 7 & 11 \\
\hline & $\%$ & $0 \%$ & $0 \%$ & $14 \%$ & $33 \%$ & $52 \%$ \\
\hline \multirow[t]{2}{*}{ D16. } & \# & 0 & 0 & 1 & 10 & 10 \\
\hline & $\%$ & $0 \%$ & $0 \%$ & $5 \%$ & $48 \%$ & $48 \%$ \\
\hline \multirow[t]{2}{*}{ D17. } & $\#$ & 0 & 0 & 1 & 7 & 13 \\
\hline & $\%$ & $0 \%$ & $0 \%$ & $5 \%$ & $33 \%$ & $62 \%$ \\
\hline
\end{tabular}

Table 6 Frequencies of the global responses of MUCSL

Figure 7 shows a bar chart with the frequency of responses from all participants.

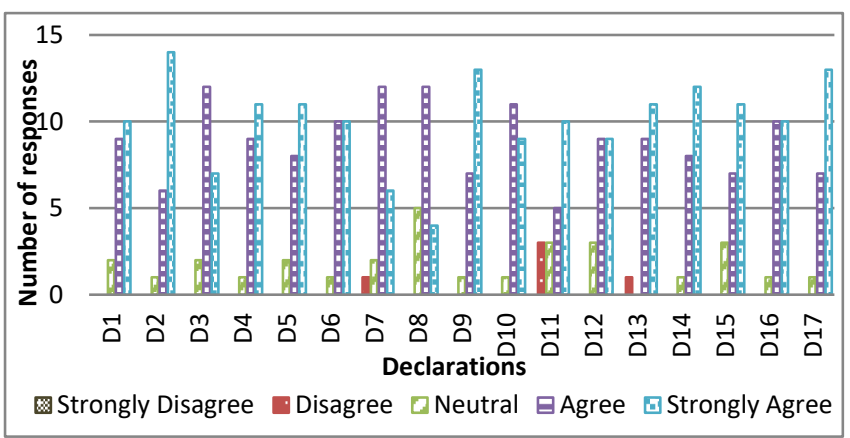

Figure 7 Overall response distribution

Figure 8 shows the responses of the different statements using a stacked bar graph by marking the percentiles.

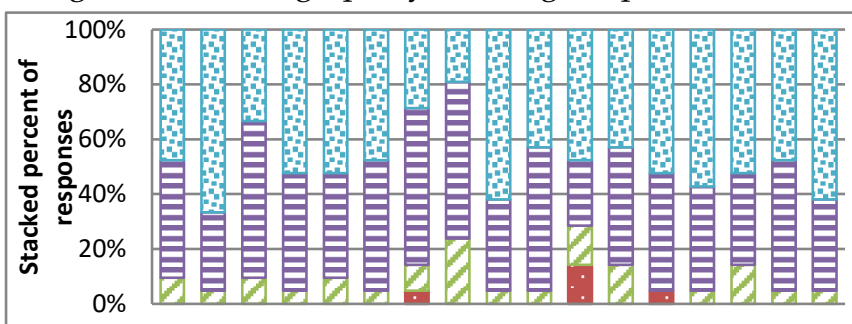

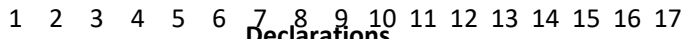
娄Strongly Disagree Disagree $\square$ Neutral $\square$ Agree $\square$ Strongly Agree

Figure 8 Stacked bar graph with the global responses

Based on the latest data shown, the following interpretations can be suggested:

- D2 has $67 \%$ of the votes as 'totally agree' and $29 \%$ as 'agree', while only $5 \%$ of the votes were 'neutral'. In number of votes, this is 14,6 and 1 , respectively. This indicates that the participants agree, at least in this statement, except for a small minority, being this the statement best valued.

- $\quad$ D9 and D17 are the following two most valued statements, changing one 'totally agree' to just 'agree'.

- On the other hand, D7 is the statement with the worst opinions and with the most different responses with $57 \%$ of the votes as 'totally agree', $29 \%$ as 'agree', $10 \%$ as 'neutral' and $5 \%$ as 'disagree'. However, D11 is the worst rated by having $48 \%$ of the votes as 'totally agree', $24 \%$ as 'agree', $14 \%$ as 'neutral' and $14 \%$ as 'disagree'.

\section{Related Work}

Currently, there is still a great lack of scientific literature on DSLs in the context of this work. In fact, some works [29] emphasize that those kinds of languages are largely non-existent, and that this is one of the areas that need to be addressed in the future.

IFTTT (If This, Then That) [46] is a proprietary Web service-based platform that aims to create sets of simple conditional statements, called applets. Such applets are triggered when a change is detected on other connected services such as social networks or emails providers. Apparently, it has a similar purpose to MUCSL. However, they may be used on different scenarios. IFTTT focuses on automate tasks (e.g., related to social networks or home automation) using graphical containers in which users only have to indicate some information requested by the container but the flexibility is limited and the structure of the possibilities is very fixed.

In addition, some of the few available works have also a different scope and objectives. For example, $\mu \mathrm{PnP}$ provides a platform-independent driver language for the IoT that enables the implementation of driver functionality in a high-level way. However, the language includes multiple options that require programming skills [47].

There are other visual languages. For example, DSL-4-IoT is a graphical modelling language, using formal presentations and abstract syntax in a metamodel to create IoT related applications [48], [49]. The PervML is another graphical DSL, created to provide developers with different elements to describe IoT systems.

Others, like the PIG DSL, focuses on a very concrete task. In this case, the definition of processes to handle the huge amount of data that is generated in the IoT in a declarative way [50].

SensApp is a platform to support cloud experiments. It uses a DSL called Gatling. It is very reminiscent of a general-purpose language due to its complexity and because of the number of possibilities it allows. For example, it is needed to create and extend classes and methods [51].

There are other works more oriented to a specific context. For example, Vitruvius [52], [53] is a platform focused on on-road vehicles and is designed to simplify the collection of information in order to generate applications quickly. Therefore, interconnections are created using a DSL between vehicles and users' mobile devices with the aim of creating applications based on the collected real-time data. 
In addition to the previous works, our research shows that very little current work is closely related to ours. However, for comparison purposes, we provide an overview of some representative works focusing on our previous work with Midgar, other middlewares and IoT platforms.

\subsection{Migar platform and Migar Object Interconnection Specific Language}

Domain-Specific Languages have been widely used in Web applications development such as HTML, CSS, and XML. In our early work [18], we have developed a platform and a graphical DSL for the users to specific connections between heterogeneous objects through a graphic interface. Users must define the interconnections among objects, which are registered in Midgar, using the DSL. It allows users to define conditions, actions, loops, sleeping times for objects, and even Java source code that involves any of the integrated objects. They can define relational operations using fixed numbers or using collected or realtime data. Thus, they must create events like 'when the temperature sensor reaches $30^{\circ} \mathrm{C}$ degrees, then...' This graphic representation is sent to the parser and then automatically transformed into applications for connecting objects.

The language reported in this paper, known as MUCSL, complements the graphical DSL and allows the users to specify object connections in natural language, rather than in a graphical one. In this case, we also present a DSL, which is a restricted language designed specifically for object connections. However, in this case, we have developed a textual DSL, which is very close to natural language. Therefore, this facilitates the automated source code generation from use cases, as long as the follow certain rules.

\subsection{Middleware}

The Web and Internet services have been used as ubiquitous middleware to facilitate the implementation of new functionality and innovative applications for smart objects [3]. Middleware is 'a software layer or a set of sub-layers interposed between the technological and the application levels' [22].

On a small scale, Mashups can be thought of as a kind of middleware that uses content from more than one source to create a single new service displayed in a single graphical interface. Mashups can be used to link event and data streams from physical objects with each other as well as with Web services [3], [54].

On a larger scale, service-oriented middlewares has been used to support interaction and integration between different technologies, applications, or communication protocols [22]. On the Internet scale, IoT middleware has begun to emerge to facilitate communication between heterogeneous and dynamic objects at different levels of abstraction and granularity [17].

Our user-oriented language can be used to aid users to specify object connections at the application level of the IoT.

\section{3 loT Platforms}

There are different platforms, which, although not being specifically a DSL, have been designed with similar objectives to those mentioned in this work.

For example, Paraimpu [55], which can integrate heterogeneous data and connect different sensors and actuators using a specific message mechanism. It allows reducing the complexity, but users still need some programming skills. In addition, it does not allow control structures. On the other hand, Midgar provides a higher level of abstractions and allows different powerful constructors like loops or timers.

Xively [56] is a supplier of cloud REST services and libraries for different platforms such as Arduino and Android. Once specific conditions are met, devices can communicate with the services to perform only an action, which can reduce the possibilities of creating complex applications. Midgar, however, does not put any limitation when creating conditions and actions, and it even allows creating nested conditions.

ThingSpeak [57] is another platform to connect services and objects by creating different channels through which data can be transmitted. It provides convenient mechanisms to display interactive charts. However, the system requires general-purpose programming skills to develop applications. Midgar, by contrast, is based on the use of a DSL, making it easier to perform the same type of operations

Nimbits has a different approach. It includes a downloadable server that can be used to create customized IoT servers with REST services. Midgar also uses a similar idea. One of the main differences between both is that Nimbits manage the concept of 'trigger', to perform computation depending on values of data. However, Nimbits can only use three parameters, while with Midgar there are no restrictions.

SIoT [58], [59], is a platform for the Social Internet of Things based on previous research [58]-[60]. As in other works, it allows creating channels to move data among devices. However, unlike Midgar, it lacks a graphical DSL to make it easier the creation of connections between the different smart objects involved. The rest of the platform is similar to ThingSpeak because SIoT is based on the core of this one.

Finally, Open.Sen.se was a platform that supports several different protocols. It also uses channels to move data and create applications based on the data. It is also possible to carry out actions when different events occur. The main drawback is that the platform only allows creating simple connections among objects and Web services. In contrast, Midgar is valid to create much more complex collaboration among objects.

Figure 7 shows the related work summarized. Here, we show the platforms according to the connection type that they allow. Machine to Web Service (M2WS) means that you only can send the data from your object to a web service and vice versa, but not from an object to another object. The column called 'Structures' means that that platform allows using any control structure of a GPL. However, some of those platforms need to allow it because you have to develop the source code and have expert programming skills using a GPL like column 'Need to develop' 
shows. The same case occurs with the column 'APIs' in the platforms that require the use of complex APIs, and 'Complex applications' in the platforms which allows to define complex behavior.

\begin{tabular}{|l|c|c|c|c|c|}
\hline Platforms & $\begin{array}{l}\text { Connection } \\
\text { Type }\end{array}$ & $\begin{array}{c}\text { Struc- } \\
\text { tures }\end{array}$ & $\begin{array}{c}\text { Need } \\
\text { to de- } \\
\text { velop }\end{array}$ & APIs & $\begin{array}{c}\text { Complex } \\
\text { applications }\end{array}$ \\
\hline Middleware & $\begin{array}{c}\text { You have to de- } \\
\text { velop it }\end{array}$ & Yes & Yes & Yes & Yes \\
\hline Paraimpu & $\begin{array}{c}\text { Textual DSL and } \\
\text { 3 parties services }\end{array}$ & No & Yes & No & Yes \\
\hline Xively & M2WS & Only & Yes & Yes & No \\
\hline ThingSpeak & Channels & Yes & Yes & Yes & Yes \\
\hline Nimbits & Forms + Trigger & No & No & No & No \\
\hline SIoT & Channels & Yes & Yes & Yes & Yes \\
\hline Open.Sen.se & M2WS & Yes & Yes & $?$ & No \\
\hline Midgar & A User-Oriented & Yes & No & Not nec- & Yes \\
\hline
\end{tabular}

Table 7 Comparison between the related works

\section{CONCLUSION AND FUtURE WORK}

This paper has presented a novel approach to enable users to specify dynamic interconnections for their IoT objects to serve their own purposes. The main contributions of this approach are summarized as follows:

First, this approach contributes to our integrated development process for the IoT-based applications, which includes registering the objects to the IoT platform, describing their interconnection in use cases, transforming the use cases into source code, and finally executing the IoT applications through the interconnected objects. This approach thus addresses the challenge to create the necessary source code for the objects automatically from the use cases, provided that they keep some small rules according to the parser.

Second, our approach provides users with a simple use case specification language MUCSL and supports automatically transformation of use cases into the source code. This allows users to connect their own objects without the need for them to learn programming languages or operating systems of the objects. Our approach thus represents a tiny step towards bringing the IoT closer to the users, and empowering users with the control and connectivity of their IoT objects.

This contribution supplements deficiencies in other works previously done and provided a programming language very close to natural language to reduce the learning curve of the users at the same time it offers a clearer interface.

We have proved the concept of this approach through developing the working prototype and demonstrated the feasibility of this approach through an experiment and a survey. The feedback from 21 participants was very positive: 19 out of 21 participants (90\%) either totally agreed or agreed that our approach will be useful and beneficial for IoT. However, our approach still needs further improvements as the remaining participants (2 out 21) who commented that users would still need to have some prior knowledge of use case specification in order to use MUCSL. Despite this concern, we believe that users can learn the basic syntax of use case specification quickly and can therefore master MUCSL easily.

Our future work will extend MUCSL to support more complex use cases so that users can specify more requirements. We will also investigate advanced NLP techniques to support the syntactic and semantic processing of the use cases. Our goal is to create a more flexible, yet powerful, language for users. Such enhancement will enable users to describe more freely what they want from IoT applications.

Our current work has not considered the optimization, scalability, and performance of the IoT connectivity, as the number of interconnected objects is currently small. With the ever-increasing number of smart objects, their interconnections will become more complex. How to reduce the coupling of many objects while maintaining the high connectivity and performance is a major challenge in our future research.

A similar situation occurs with the security because the use of the Midgar platform provides as a secure system to send the messages that have been published in [32]. However, one of the improvements and future work is the improvement and implementation of a secure registration. Besides, currently, Midgar is an open system that allows accessing to all objects. Then, it is required the implementation of roles for users to create a permission system according to the necessities of people and objects.

As implemented right now the platform only transforms the source code defined with the DSL to final working code when it is correct and there are not any mistake. However, we plan to adjust the definition of the language to be compatible with the Language Server Protocol (LSP)2, an open, JSON-RPC-based protocol used between integrated development environments and servers that provide programming language-specific features. That will open a number of possibilities to create custom features such as syntax user-defined colouring, semantic colouring, error checking, auto-completion, formatting, hover information, mark occurrences, rename refactoring, debugging or quick fix proposals among others.

Another possible future work line could be the introduction of different protocols and data formats (JSON, $\mathrm{CSV}$, etc.) to allow users to choose between the protocol and format that they want or prefer to use.

\section{ACKNOWLEDGEMENT}

This work was performed by the 'Ingeniería Dirigida por Modelos MDE-RG' research group at the University of Oviedo under Contract No. FC-15-GRUPIN14-084 of the research project 'Ingeniería Dirigida Por Modelos MDE$R G^{\prime}$. The project was funded by the Government of the Principality of Asturias. The work was also supported by 
the economic mobility grants of the University of Oviedo, which enabled the third author to visit the University of Manchester in 2013.

\section{REFERENCES}

[1] G. Santucci, "The internet of things: Between the revolution of the internet and the metamorphosis of objects," 2010.

[2] International Telecommunication Union, "The Internet of Things," 2005.

[3] F. Mattern and C. Floerkemeier, "From the Internet of Computers to the Internet of Things," in Lecture Notes in Computer Science (including subseries Lecture Notes in Artificial Intelligence and Lecture Notes in Bioinformatics), vol. 6462 LNCS, Springer, Berlin, Heidelberg, 2010, pp. 242259.

[4] J. Cheng, J. Cheng, M. Zhou, F. Liu, S. Gao, and C. Liu, "Routing in Internet of Vehicles: A Review," IEEE Trans. Intell. Transp. Syst., vol. 16, no. 5, pp. 2339-2352, Oct. 2015.

[5] Z. Ning et al., "A Cooperative Quality-Aware Service Access System for Social Internet of Vehicles," IEEE Internet Things J., vol. 5, no. 4, pp. 2506-2517, Aug. 2018.

[6] W. Xu et al., "Internet of vehicles in big data era," IEEE/CAA J. Autom. Sin., vol. 5, no. 1, pp. 19-35, Jan. 2018.

[7] C. González García, D. Meana-Llorián, B. C. P. G-Bustelo, and J. M. C. Lovelle, "A review about Smart Objects, Sensors, and Actuators," Int. J. Interact. Multimed. Artif. Intell., vol. 4, no. 3, pp. 7-10, 2017.

[8] K. Ashton, "That 'Internet of Things' thing," RFiD J., vol. 22 no. 7, pp. 97-114, 2009.

[9] G. Cueva-Fernandez, J. Pascual Espada, V. García-Díaz, and R. Gonzalez-Crespo, "Fuzzy decision method to improve the information exchange in a vehicle sensor tracking system," Appl. Soft Comput., vol. 35, pp. 1-9, Oct. 2015.

[10] N. Taušan, J. Markkula, P. Kuvaja, and M. Oivo, "Choreography in the embedded systems domain: A systematic literature review," Inf. Softw. Technol., vol. 91, pp. 82-101, Nov. 2017.

[11] M. Faheem and V. C. Gungor, "Energy efficient and QoSaware routing protocol for wireless sensor network-based smart grid applications in the context of industry 4.0," Appl. Soft Comput. J., 2017.

[12] B. Zeng and Y. Dong, "An improved harmony search based energy-efficient routing algorithm for wireless sensor networks," Appl. Soft Comput. J., vol. 41, pp. 135-147, 2016.

[13] E. Lee, Y.-G. Kim, Y.-D. Seo, K. Seol, and D.-K. Baik, "RINGA: Design and verification of finite state machine for self-adaptive software at runtime," Inf. Softw. Technol., vol. 93, no. September 2016, pp. 200-222, Jan. 2018.

[14] N. M. do Nascimento and C. J. P. de Lucena, "FloT: An agentbased framework for self-adaptive and self-organizing applications based on the Internet of Things," Inf. Sci. (Ny)., vol. 378, pp. 161-176, 2017.

[15] J. Carretero and J. D. García, "The Internet of Things: connecting the world," Pers. Ubiquitous Comput., vol. 18, no. 2, pp. 445-447, Feb. 2014

[16] Y. Sun, R. Bie, P. Thomas, and X. Cheng, "Theme issue on advances in the Internet of Things: identification, information, and knowledge," Pers. Ubiquitous Comput., vol. 19, no. 7 , pp. 985-987, 2015.

[17] K. Gama, L. Touseau, and D. Donsez, "Combining heterogeneous service technologies for building an Internet of Things middleware," Comput. Commun., vol. 35, no. 4, pp. 405-417, Feb. 2012

[18] C. González García, C. P. García-Bustelo, J. P. Espada, and G. Cueva-Fernandez, "Midgar: Generation of heterogeneous objects interconnecting applications. A Domain Specific Language proposal for Internet of Things scenarios," Comput. Networks, vol. 64, no. C, pp. 143-158, Feb. 2014.

[19] Y. Zhang, Y. Xiang, X. Huang, X. Chen, and A. Alelaiwi, "A matrix-based cross-layer key establishment protocol for smart homes," Inf. Sci. (Ny)., vol. 429, pp. 390-405, 2018. J. I. R. Molano, J. M. C. Lovelle, C. E. Montenegro, J. J. R.
Granados, and R. G. Crespo, "Metamodel for integration of Internet of Things, Social Networks, the Cloud and Industry 4.0," J. Ambient Intell. Humaniz. Comput., pp. 1-15, Feb. 2017.

O. Vermesan and P. Friess, Internet of Things: Converging Technologies for Smart Environments and Integrated Ecosystems. Aalborg, Denmark: River Publishers, 2013.

L. Atzori, A. lera, and G. Morabito, "The Internet of Things: A survey," Comput. Networks, vol. 54, no. 15, pp. 2787-2805, 2010.

I. Approach et al., "Towards Multi-layer Interoperability of Heterogeneous loT Platforms: The INTER-IoT Approach," in Integration, Interconnection, and Interoperability of IoT Systems, no. August 2017, R. Gravina, C. E. Palau, M. Manso, A. Liotta, and G. Fortino, Eds. Cham: Springer International Publishing, 2018, pp. 199-232.

B. Molina, C. E. Palau, G. Fortino, A. Guerrieri, and C. Savaglio, "Empowering smart cities through interoperable Sensor Network Enablers," in 2014 IEEE International Conference on Systems, Man, and Cybernetics (SMC), 2014, vol. 2014-Janua, no. January, pp. 7-12.

R. Casadei, G. Fortino, D. Pianini, W. Russo, C. Savaglio, and M. Viroli, "Modelling and simulation of Opportunistic loT Services with Aggregate Computing," Futur. Gener. Comput. Syst., vol. 91, pp. 252-262, Feb. 2019.

H. Cervantes and R. S. Hall, "Autonomous adaptation to dynamic availability using a service-oriented component model," in Proceedings. 26th International Conference on Software Engineering, 2004, vol. 3, no. October, pp. 614623.

S. J. Bolaños Castro, R. González Crespo, and V. H. Medina García, "Patterns of Software Development Process," Int. J. Interact. Multimed. Artif. Intell., vol. 1, no. 4, p. 33, 2011.

I. Jacobsson, M. Christersson, P. Jonsson, and G. Övergaard, Object-oriented software engineering: $A$ usecase driven approach. Addison-Wesley, 1992.

J. Mineraud, O. Mazhelis, X. Su, and S. Tarkoma, "A gap analysis of Internet-of-Things platforms," Comput. Commun., vol. 89, pp. 5-16, 2016.

C. González García, "MIDGAR: Interoperability of objects in the Internet of Things scenario using Model-Driven Engineering," J. Ambient Intell. Smart Environ., vol. 9, no. 6, pp. 799-801, Nov. 2017.

C. González García, J. P. Espada, E. R. N. Valdez, and V. García-Díaz, "Midgar: Domain-Specific Language to Generate Smart Objects for an Internet of Things Platform," in 2014 Eighth International Conference on Innovative Mobile and Internet Services in Ubiquitous Computing, 2014, pp. 352-357.

G. Sánchez-Arias, C. González García, and B. C. Pelayo GBustelo, "Midgar: Study of communications security among Smart Objects using a platform of heterogeneous devices for the Internet of Things," Futur. Gener. Comput. Syst., vol. 74, no. September, pp. 444-466, 2017.

D. Meana-Llorián, C. González García, B. C. Pelayo GBustelo, J. M. Cueva Lovelle, and N. Garcia-Fernandez, "loFClime: The fuzzy logic and the Internet of Things to control indoor temperature regarding the outdoor ambient conditions," Futur. Gener. Comput. Syst., vol. 76, pp. 275284, Nov. 2017.

[34] C. G. García, E. Núñez-Valdez, V. García-Díaz, C. Pelayo GBustelo, and J. M. Cueva-Lovelle, "A Review of Artificial Intelligence in the Internet of Things," Int. J. Interact. Multimed. Artif. Intell., vol. InPress, no. InPress, p. 1, 2018.

[35] C. González García, D. Meana-Llorián, B. C. Pelayo GBustelo, J. M. Cueva Lovelle, and N. Garcia-Fernandez, "Midgar: Detection of people through computer vision in the Internet of Things scenarios to improve the security in Smart Cities, Smart Towns, and Smart Homes," Futur. Gener. Comput. Syst., vol. 76, pp. 301-313, Nov. 2017.

[36] S. Ruby, D. Thomas, and D. H. Hansson, Agile Web Development with Rails, 3rd ed. 2008.

[37] C. Zapponi, "GitHut," 2014. [Online]. Available: http://githut.info/. [Accessed: 02-Feb-2016].

[38] TIOBE Software BV, "TIOBE Index," 
http://www.tiobe.com/index.php/content/paperinfo/tpci/index .html, $2014 . \quad$ [Online]. Available: http://www.tiobe.com/index.php/content/paperinfo/tpci/index .html. [Accessed: 02-Feb-2016].

[39] L. Eshkevari, F. Dos Santos, J. R. Cordy, and G. Antoniol "Are PHP applications ready for Hack?," in 2015 IEEE 22nd International Conference on Software Analysis, Evolution, and Reengineering (SANER), 2015, pp. 63-72.

[40] H. Zhao et al., "The HipHop compiler for PHP," ACM SIGPLAN Not., vol. 47, no. 10, p. 575, Nov. 2012.

[41] Facebook, "Hack," 2018. [Online]. Available: https://hacklang.org/. [Accessed: 17-Nov-2018].

[42] R. Likert, "A technique for the measurement of attitudes," Arch. Psychol., vol. 22, pp. 1-55, 1932.

[43] Blacksun Software, "Mousotron," 2016. [Online]. Available: http://www.blacksunsoftware.com/mousotron.html.

[Accessed: 25-Jul-2016].

[44] G. Fortino, C. Savaglio, and M. Zhou, "Toward opportunistic services for the industrial Internet of Things," in 2017 13th IEEE Conference on Automation Science and Engineering (CASE), 2017, vol. 2017-Augus, pp. 825-830.

[45] M. Kasunic, Designing an effective survey. Pittsburgh, 2005.

[46] B. Ur, E. McManus, M. Pak Yong Ho, and M. L. Littman, "Practical trigger-action programming in the smart home," in Proceedings of the 32nd annual ACM conference on Human factors in computing systems - CHI '14, 2014, pp. 803-812.

[47] F. Yang, N. Matthys, R. Bachiller, S. Michiels, W. Joosen, and D. Hughes, " $\mu \mathrm{PnP}$ : plug and play peripherals for the internet of things," in Proceedings of the tenth European conference on computer systems, 2015, p. 25.

[48] E. Azadi Marand, E. Azadi Marand, and M. Challenger, "DSML4CP: A Domain-specific Modeling Language for Concurrent Programming," Comput. Lang. Syst. Struct., vol. In Press, 2015.

[49] A. Salihbegovic, T. Eterovic, E. Kaljic, and S. Ribic, "Design of a domain specific language and IDE for Internet of things applications," in Information and Communication Technology, Electronics and Microelectronics (MIPRO), 2015 38th International Convention on, 2015, pp. 996-1001.

[50] A. F. Gates et al., "Building a High-Level Dataflow System on top of Map-Reduce: The Pig Experience," Proc. VLDB EndowmentVldb '09, pp. 1-12, 2009.

[51] S. Mosser, F. Fleurey, B. Morin, F. Chauvel, A. Solberg, and I. Goutier, "Sensapp as a reference platform to support cloud experiments: From the internet of things to the internet of services," in Symbolic and Numeric Algorithms for Scientific Computing (SYNASC), 2012 14th International Symposium on, 2012, pp. 400-406.

[52] G. Cueva-Fernandez, J. P. Espada, V. García-Díaz, C. González García, and N. Garcia-Fernandez, "Vitruvius: An expert system for vehicle sensor tracking and managing application generation," J. Netw. Comput. Appl., vol. 42, pp. 178-188, Jun. 2014

[53] V. García-Díaz, J. P. Espada, and G. C. Fernández, "Vitruvius: Vehicle sensor based model-driven engineering application generation," J. Ambient Intell. Smart Environ., vol. 10, no. 1, 2018.

[54] D. Guinard, V. Trifa, T. Pham, and O. Liechti, "Towards physical mashups in the Web of Things," in 2009 Sixth International Conference on Networked Sensing Systems (INSS), 2009, pp. 1-4.

[55] A. Pintus, D. Carboni, and A. Piras, "Paraimpu: a platform for a social web of things," in International World Wide Web Conference Com- mittee (IW3C2), 2012, pp. 401-404.

[56] LogMeln, "Xively," 2013. [Online]. Available: https://xively.com/. [Accessed: 29-Jul-2015].

[57] loBridge, "Thingspeak," 2013. [Online]. Available: http://www.thingspeak.com. [Accessed: 29-Jul-2015].

[58] L. Atzori, A. lera, and G. Morabito, "SloT: Giving a Social Structure to the Internet of Things," IEEE Commun. Lett., vol. 15, no. 11, pp. 1193-1195, Nov. 2011.

[59] R. Girau, M. Nitti, and L. Atzori, "Implementation of an Experimental Platform for the Social Internet of Things," in 2013 Seventh International Conference on Innovative Mobile and Internet Services in Ubiquitous Computing, 2013, pp.
[60] L. Atzori, A. Iera, G. Morabito, and M. Nitti, "The Social Internet of Things (SloT) - When social networks meet the Internet of Things: Concept, architecture and network characterization," Comput. Networks, vol. 56, no. 16, pp. 3594-3608, Nov. 2012. 